\title{
OCCURRENCE OF NEISSERIA OTHER THAN THE GONOCOCCUS IN THE GENITAL TRACT*
}

BY

\author{
A. E. WILKINSON
}

The Whitechapel Clinic, London Hospital

Cultural methods are now widely used for the diagnosis of gonorrhoea, and a positive culture offers the only incontrovertible proof of the diagnosis. The bacteriological criteria for the identification of the gonococcus are clearly defined, but in routine work they are not always stringently applied. An organism with an oxydase positive colony which microscopical examination shows to be a Gram-negative diplococcus is often accepted as a gonococcus without further investigation. Many workers have reported the finding of other Neisseria besides the gonococcus in the genital tract, but there is little information as to the frequency with which these organisms are found and thus the size of the diagnostic problem they present is uncertain. To obtain this information an attempt was made to carry out a full identification of all Neisseria found in cultures from patients at their first attendance at the Whitechapel Clinic. Specimens from presumably healthy pregnant women have also been examined.

\section{Technique}

The gonococcus is seldom the predominant organism in cultures from female patients. Careful plating out of inocula so as to get discrete colonies is an important prerequisite for its isolation in pure culture. The following method has been found to give satisfactory results.

Specially treated swabs are moistened with the secretion to be cultured and the swabs are placed in Stuart's transport medium (Moffett, Young, and Stuart, 1948), and placed in the refrigerator until they can be conveniently plated out. A preliminary survey on 204 patients showed that gonococci could be isolated from swabs left for periods up to $52 \mathrm{hrs}$. in this medium (Wilkinson, 1951). The nutrient medium used was McLeod's chocolate agar containing 10 per cent. horse

* Received for publication September 1, 1951. blood. Inoculated plates were incubated for $24 \mathrm{hrs}$. at $35^{\circ}-36^{\circ} \mathrm{C}$. in an atmosphere containing $\mathrm{CO}_{2}$, produced by burning a candle in a closed container. This was followed by a further $24 \mathrm{hrs}$. in the ordinary atmosphere. Suspicious colonies were picked off and stained films examined microscopically. Those having the appearance of Neisseria were subcultured on to hydrocele agar slopes. The primary cultures were finally flooded with oxydase reagent (freshly prepared 1 per cent. aqueous dimethyl-p-phenylenediamine $\mathrm{HC1}$ ) to ensure that the colonies under examination were oxydase positive and that no potential Neisseria had been missed. Slide agglutination tests were also carried out on Neisseria colonies from the primary plates; the results of this part of the investigation will be reported separately.

After $24 \mathrm{hrs}$. incubation in a partial $\mathrm{CO}_{2}$ atmosphere the hydrocele agar subcultures were examined microscopically for purity and, if satisfactory, were subcultured on to sugar media. These consisted of 1 per cent. glucose, maltose, and saccharose in 20 per cent. hydrocele agar at a $\mathrm{pH}$ of $7 \cdot 4$, with phenol red as indicator. They were distributed in $3 \mathrm{ml}$. amounts in screw-capped bijou bottles. After incubation, Gram stained films of any culture showing the production of acid were examined to confirm the purity of the growth. Whenever the fermentation reactions were anomalous, they were checked by repetition. It was found to be important that the screw caps of the bijou bottles should only be screwed on loosely. If they were tightened to give an airtight joint, the fermentation reactions were often anomalous in that there was apparent slight acid production in the maltose and saccharose bottles by organisms which subsequently proved to be gonococci. If such bottles were reincubated after the caps had been loosened, the $p H$ gradually became alkaline, except in the case of glucose. It seems probable that in the airtight bottle sufficient $\mathrm{CO}_{2}$ may accumulate to change the colour of the indicator.

Organisms which, on examination of the primary culture had the colonial and microscopical appearance of gonococci and were oxydase positive, were reported as Presumptive Gonococci. If isolation in pure culture and confirmation by fermentation reactions were achieved, a supplementary report to this effect was made. 


\section{Clinical Material Examined}

This fell into three categories :

Group A.-204 female patients in whom cervical secretions only were examined by the method outlined above. Cultures were made from the urethral, cervical, vaginal, and rectal secretions of a further 891 consecutive patients at their first attendance at the clinic. The total in the group was therefore 1,095 .

Group B.-The urethral secretion of 130 men complaining of urethral discharge was examined. These patients were not examined consecutively, but no attempt was made to select any particular type of case.

Group C.- The urethral and cervical secretions of 350 patients attending an antenatal clinic were examined.

\section{Results}

The results of the examination of these patients are summarized in the following Table :

TABLE

FREQUENCY OF ISOLATION OF NEISSERIA

\begin{tabular}{|c|c|c|c|c|}
\hline \multicolumn{2}{|l|}{ Group } & A & B & $\mathrm{C}$ \\
\hline \multicolumn{2}{|l|}{ Patients in group } & 1095 & 130 & 350 \\
\hline \multicolumn{2}{|c|}{$\begin{array}{l}\text { Patients in whom Neisseria } \\
\text { were found }\end{array}$} & 290 & 68 & 2 \\
\hline \multirow{2}{*}{$\begin{array}{l}\text { Gonococci (fully } \\
\text { identified) }\end{array}$} & No. & 251 & 63 & - \\
\hline & $\% *$ & $86 \cdot 6$ & $92 \cdot 6$ & \\
\hline \multirow{2}{*}{$\begin{array}{l}\text { Presumptive } \\
\text { Gonococci } \dagger\end{array}$} & No. & 29 & 4 & - \\
\hline & $\% *$ & $10 \cdot 0$ & $5 \cdot 9$ & \\
\hline \multirow{2}{*}{$\begin{array}{l}\text { Neisseria other } \\
\text { than Gonococci }\end{array}$} & No. & 10 & 1 & 2 \\
\hline & $\% *$ & $3 \cdot 4$ & $1 \cdot 5$ & \\
\hline
\end{tabular}

* Percentages are calculated from the total number of Neisseria found in each group.

t These strains were lost in subculture so that full identification was not obtained.

The thirteen strains of Neisseria which proved not to be gonococci were investigated as to their fermentation reactions, pigment production, colonial morphology, and ability to grow on plain agar. Five strains were identified as $N$. catarrhalis, six as $N$. flava, and two as $N$. sicca.

\section{Case Reports}

Brief clinical histories of patients who were harbouring some of these organisms are as follows :
Case 1, H 13925, Female, aged 46.-On August 23, 1950 , she attended the clinic complaining of vaginal discharge of 3 months' duration. History of gonorrhoea in 1942 with satisfactory tests of cure. No Neisseria seen in urethral or cervical smears; cultures from urethra and vagina showed a moderate growth of $N$. catarrhalis. Gonococcal complement-fixation test negative. She failed to attend again until September 30, 1950, when she no longer complained of any discharge. Smears negative, but urethral and vaginal cultures again gave a heavy growth of $N$. catarrhalis, which was the predominant organism. No treatment was given and she was discharged from observation on October 18, 1950.

Case 2, H 7615, Female, aged 28.-On November 15, 1950 , she attended the clinic complaining of a vaginal discharge of 2 months' duration. There was a history of gonorrhoea and trichomonas infection in 1947 when tests of cure had been satisfactory. Smears negative but urethral culture showed a few colonies of $N$. flava. Gonococcal complement-fixation test negative. Trichomonads present in vaginal secretions. The patient is under observation but no more Neisseria have been isolated.

Case 3, H 14499, Female, aged 28.-This patient was examined as her husband had been found to have general paralysis of the insane. She had cervicitis and a slight vaginal discharge. Smears negative; a single colony of $N$. sicca found on urethral culture. Gonococcal complement-fixation test negative.

Case 4, H 14937, Female, aged 28.-This patient was treated for gonorrhoea on four occasions between September, 1948, and July, 1949 . She attended the clinic again in March, 1951, complaining of a vaginal discharge for the past 3 weeks. Smears negative. $N$. catarrhalis grown from the cervix. Gonococcal complement-fixation test negative.

Case 5, H 15259, Female, aged 34.-This patient attended the clinic because her consort was attending another hospital for a urethral discharge. On clinical grounds this was considered to be due to gonorrhoea and although smears and cultures were negative, he had been treated with penicillin. Her smears were negative but a moderate growth of $N$. catarrhalis was obtained on cultures from the urethra, cervix, and vagina. Gonococcal complement-fixation test negative. Trichomonads found in vaginal secretions. Local treatment given. No Neisseria found in subsequent cultures.

Case 6, H 14191, Female, aged 36.-This patient came to the clinic with a complaint of slight vaginal discharge on October 13, 1950. She had previously been treated for gonorrhoea on four occasions in 1944. No suspicious organisms found in smears but moderate numbers of Neisseria present in cultures from urethra, cervix, and vagina. These simulated the gonococcus sufficiently closely for a report of Presumptive Gonococci to be made. Further investigation showed that two strains of Neisseria were present; these were identified as $N$. catarrhalis and $N$. flava. Gonococcal complementfixation test negative. On the basis of the erroneous report treatment with penicillin was given. Neisseria 
were not isolated again in cultures taken over a period of 8 months.

Case 7, H 14041, Female, aged 46.-This patient came to the clinic complaining of irritation in the anal region for the previous 2 days. Clinically there was no proctitis. Smears from the urethra, cervix, and rectal mucosa were negative. A culture from the rectal mucosa grew coliform bacilli and a single colony of N. sicca.

Case 8, T 46874, Male, aged 38. - This man complained of a urethral discharge of 2 days' duration. His last exposure to infection had been 12 days before the onset of the discharge, which was muco-purulent in character. Smears showed large Gram negative diplococci, and a culture grew Staph. albus, streptococci, and a single colony of $N$. sicca. Gonococcal complement-fixation test negative. Treatment with sulphatriad was begun but the patient did not attend again. It was not possible to trace his consort.

\section{Discussion}

The occurrence in the genital tract of other members of the Neisseria besides the gonococcus has been known for many years. The early literature has been discussed by Thomson (1923), who also reported his own investigations, and more recently by Harkness (1950). Some of the early descriptions of Neisseria are not clear, and our knowledge of the non-pathogenic members of the group is still incomplete. Topley and Wilson (1946) feel that, with the possible exception of $N$. catarrhalis, other saphrophytic types should be combined into a single species for which they suggest the name $N$. pharyngis.

Carpenter and Charles (1942) reported the finding of seven strains of Group 1 meningococci among 103 strains of Gram negative diplococci isolated from the genital tract. Six came from men with anterior urethritis (one also had epididymitis) and the seventh from a woman with chronic cervicitis. No other meningococci had been cultured from the genital tract during the previous decade in their laboratory. Gonococci had been isolated from one of the patients one month before the finding of meningococci ; in a second, the strain fermented only glucose when first isolated, but, after preservation in the frozen state for an unspecified time, was found to ferment maltose in addition. Organisms cultured from the same patient 70 days later were found to be gonococci. The other five strains were examined only after preservation for a month at $-80^{\circ} \mathrm{C}$. Four of these patients had been treated with sulphonamides before the cultures were taken and the authors point out the possibility has to be considered that this factor, or the maintenance of strains at a low temperature, may have altered their biochemical and serological behaviour. Murray
(1939) has also discussed the occurrence of meningococci in the male urogenital tract.

Meningococci are also occasionally found in cases of conjunctivitis and the suspicion of gonococcal ophthalmia may be roused until final identification of the organism is obtained. One such case has been encountered in this laboratory and others have been reported recently by Stuart and McWalter (1948).

Carpenter (1943) reported the isolation of $N$. flava from three out of 505 patients who were investigated for evidence of gonorrhoea. In one male case, the urethral discharge showed Gram negative diplococci, slightly more spherical than gonococci. Stained films from the other two patients, who had cervicitis, were negative. Coutts and Barthet (1936) also described the finding of $N$. flava in the cervical secretions of two prostitutes.

Weaver (1950) examined 1,014 female inmates of a mental institution. Gram negative intra- and extra-cellular diplococci were found in the secretions of twelve girls with vulvovaginitis. These organisms were identified as $N$. sicca.

Wax (1949) has reported the largest series of investigations into the genital Neisseria. He examined 790 cultures in which Neisseria had been found. These came from patients who had been treated for gonorrhoea with penicillin. About 60 per cent. of the strains were isolated in pure culture and identified by means of fermentation reactions. Eighty-three strains (19 per cent.) out of 442 tested proved not to be gonococci. In a smaller series of positive cultures taken before treatment was instituted from 61 female and 26 male patients, 79 strains gave the sugar reactions of the gonococcus while eight did not. In a second paper (Wax, 1950), the results were given of a more detailed study of 57 of these non-gonococcal strains. Thirty-seven were identified as $N$. sicca, although none had the classical rough colonies of this organism, seven were $N$. flava, three $N$. subflava, three $N$. catarrhalis, and one $N$. meningitidis. The remaining five strains belonged to miscellaneous groups. Johnson (1951) found 31 strains of $N$. sicca and twelve of $N$. subflava during the routine examination of cultures for gonococci taken both before and after treatment. She noted that these organisms are slower in giving a positive oxydase reaction than is the gonococcus, the colouration first appearing at the periphery of the colony. De Bord (1942) and Hendriksen (1947) have described the finding of organisms in the genital tract which, although they are oxydase positive and may assume the diplococcal form, are not Neisseria. The latter author has also isolated atypical strains of $N$. catarrhalis from the genital tract (Hendriksen, 1945). 
It has been noted during this work that some strains of gonococci ferment glucose much more rapidly than others. The great majority produce enough acid in 18 to 24 hrs. to cause a definite change in colour of the indicator (phenol red). Among 193 strains where this point was specially noted there were nine which did not produce demonstrable acid until 48 hrs. after inoculation, even though there was a good growth after 18 to 24 hours. On subculture, these slow-fermenting strains rapidly acquired the power of acid production within $24 \mathrm{hrs}$. of inoculation. No difference in colonial appearance was noticed between the two groups. This phenomenon has been described by Reyn (1948); she observed differences between the colonies of the two types but found that the slow fermenters were not serologically distinct. Bahn and others (1945) reported that the fermentation of glucose is delayed in cultures of gonococci which have been made resistant to penicillin in vitro. In two of Reyn's cases atypical strains were also found in the patients' contacts. In one female patient in the present series slow-fermenting strains were isolated on two occasions separated by an interval of 10 months. As she had been re-exposed to infection during the interval, it was not possible to decide whether the second isolation of slow-fermenting organisms represented a relapse of the original infection or a reinfection.

The work presented in this paper confirms the reports of other workers that Neisseria other than the gonococcus may be found in cultures from the genital tract. 3.4 per cent. of the Neisseria isolated from female patients attending the Whitechapel Clinic were not gonococci; in male patients the figure was 1.5 per cent. It should be remembered, however, that cultures were taken from four sites in the majority of the female patients, whereas in the males only the urethral secretion was cultured. In most cases these organisms are scanty but in two cases where $N$. catarrhalis was grown they were the predominant organism in the culture. They appear to be harmless commensals whose only importance lies in their superficial resemblance to the gonococcus. They were only once seen in stained films and were easily distinguishable from the gonococcus. The colonial appearance under a low magnification (+6 lens) is usually sufficiently distinctive to prevent confusion provided that the colonies are examined before the application of the oxydase reagent. The colour develops slowly and is usually less dense than is found in gonococcus colonies. In stained films of gonococci from 48-hr.-old colonies there is nearly always some autolysis to be seen with variation in the intensity of staining of the cocci. In contrast to this, the individual members of these other Neisseria stain much more evenly and more intensely and do not show this tendency to autolysis which is one of the most characteristic appearances of the gonococcus, although it is also seen in meningococci.

Although these non-gonococcal Neisseria have not been isolated as often as in the series reported by Wax $(1949,1950)$, it seems certain that they constitute a possible source of error in the bacteriological diagnosis of gonorrhoea. Any laboratory which undertakes cultural examinations for the gonococcus should be prepared to attempt its full identification by fermentation reactions and should not be content with any less stringent criteria.

\section{Summary}

(1) Full identification of Neisseria found in cultures from 1,575 patients has been attempted.

(2) 3.4 per cent. of Neisseria isolated from female patients attending a Venereal Diseases Clinic were found not to be gonococci. The corresponding figure in male patients was 1.5 per cent.

This work was carried out during the tenure of an Insole Scholarship awarded by the British Medical Association.

\section{REFERENCES}

Bahn, J. M., Ackerman, H., and Carpenter, C. M. (1945). Proc. Soc. exp. Biol., N.Y., 58, 21.

Carpenter, C. M. (1943). Amer. J. pub. Health, 33, 135. , and Charles, R. (1942). Amer. J. publ. Hlth, 32, 640.

Coleman, M. B. (1946). J. vener. Dis. Inform., 27, 159.

Coutts, W. E., and Barthet, O. D. (1936). British Journal of Venereal Diseases, 12, 75.

De Bord, G. G. (1942). Iowa St. Coll. J. Sci., 16, 471. Harkness, A. H. (1950). " Non-gonococcal Urethritis". Livingstone, Edinburgh.

Henriksen, S. D. (1946). Acta derm. venereol., Stockh., 26, 506.

(1947). Acta path. microbiol. scand., 24, 184.

Johnson, J. (1951). Amer. J. Syph., 35, 79.

Moffett, M., Young, J. L., and Stuart, R. D. (1948). Brit. med. J., ii. 421.

Murray, E. G. D. (1939). Urol. cutan. Rev., 43, 739.

Reyn, A. (1948). Acta. Derm. venereol., Stockh., $28,381$.

Stuart, R. D., and McWalter, D. (1948). Lancet, 1, 246.

Thomson, D. (1923). " Gonorrhoea ". Oxford Medical

Publications. with contributions by Lees, D., Mills,

C. H., Thomson, R., and Maclachlan, K.

Wax, L. (1949). J. vener. Dis. Inform., 30, 145. -(1950). Ibid., 31, 208.

Weaver, J. D. (1950). Amer. J. Obstet. Gynec., 60, 257.

Wilkinson, A. E. (1951). British Journal of Venereal Diseases, 27, 200.

Wilson, G. S., and Miles, A. A. (1946). " Topley and Wilson's Principles of Bacteriology and Immunity", 3rd. ed., rev. G. S. Wilson and A. A. Miles. Arnold, London. 